\title{
Statistical study of the proton isotropy boundary
}

\author{
E. A. Lvova, V. A. Sergeev, and G. R. Bagautdinova \\ Institute of Physics, St. Petersburg State University, St. Petersburg, Russia \\ Received: 5 October 2004 - Revised: 4 February 2005 - Accepted: 8 February 2005 - Published: 3 June 2005
}

\begin{abstract}
Based on a large data set of polar NOAA-type satellite observations we studied the latitude-MLT shape of the $80 \mathrm{keV}$ proton isotropy boundary (IB) as a function of the solar wind parameters and magnetic activity. Using "snapshots" of isotropy boundaries near-simultaneously crossed at four points we found that its equatorward expansion, as well as its dawn-dusk shift, depends mostly on the $A E$ index and on the corrected $D_{s t}{ }^{*}$, whereas the amplitude of the IB daily variation is mostly controlled by the solar wind dynamic pressure. Applying a nonlinear, multi-parametric, least-square regression procedure, the empirical relationship describing the IB latitude as a function of MLT and $A E, \mathrm{Pd}$, $D_{s t}{ }^{*}$ parameters was obtained. Comparing it with the predictions from the Tsyganenko-2001 model we found a good agreement during the quiet time but some important differences during the disturbed periods. Interpretation of these results in terms of the properties of the magnetospheric configuration is briefly discussed.
\end{abstract}

Keywords. Magnetospheric physics (Energetic particles, Precipitating; Energetic particles, trapped; Solar windmagnetosphere interactions)

\section{Introduction}

A typical observation of a low-altitude polar satellite crossing the auroral oval is the anisotropic (loss cone is nearly empty) energetic proton fluxes at subauroral latitudes, which change sharply at the isotropic flux distribution (in the loss cone) in the poleward part of the oval as a satellite moves poleward. This feature of particle fluxes is observed at all magnetic local times (MLT) and for all magnetospheric conditions. This sharp boundary (isotropy boundary, IB) was interpreted as the ionospheric mapping of the transition between the regions of adiabatic and nonadiabatic proton motion in the equatorial current sheet (Sergeev et al., 1983) or in the outer dayside cusp (Sergeev et al., 1997), when the ratio between magnetic field curvature radius $R_{c}$ and the proton gyroradius $\rho$ decreases below the threshold $R_{c} / \rho \sim 8$, see

Correspondence to: E. A. Lvova

(kat@geo.phys.spbu.ru)
Sergeev et al. (1988); West et al. (1978), particularly in the current sheet

$R_{c} / \rho=B_{n}^{2} /\left(G \partial B_{t} / \partial n\right)$.

Here $n$ and $t$ denote the normal and tangential directions with respect to the current sheet, and $G=m v / q$, where $G, m, v$ and $q$ are the rigidity, mass, velocity and charge of the measured particles, accordingly. This threshold condition allows one to find the isotropic boundaries in any magnetospheric model. For the protons with energies of $\sim 80 \mathrm{keV}$ the transition between adiabatic and nonadiabatic behavior on the nightside occurs at the distance $5-9 R_{E}$ at the equator, see Fig. 1a.

Different ways of using the IB as an indicator of the magnetic field configuration were discussed in the literature. A special algorithm (IBA) was suggested in Sergeev et al. (1988) to specify the version of the Tsyganenko model and to sense remotely the nightside equatorial magnetic field based on the IB position. The magnetic field at geostationary orbit evaluated with this method was in good agreement (correlation coefficient $r \sim 0.9$ ) with the real magnetic field observed at the geostationary GOES spacecraft (Sergeev et al., 1993); (Newell et al., 1998), showing that the IB latitude is effectively controlled by the tail magnetic field. A simple index of magnetotail stretching (MT-index) was proposed for tail current monitoring to perform the accurate mapping from the ionosphere to the magnetosphere (Sergeev et al., 1995, later referred to as SG95). The global IB shape (its CGLat dependence on MLT), as well as its dependences on the solar wind dynamic pressure Pd and magnetic activity index $A E$ was first evaluated in SG95, although the available database was rather small (one month of data obtained from 2 spacecraft). Sergeev et al. (1997) considered dayside isotropic precipitation and showed that a dawnside shift of the IB symmetry line was observed. This shift was shown to depend on the IMF $B_{z}$ component and the $A E$-index: the increase of activity causes both the clockwise rotation of the symmetry line and the decrease in the IB latitude. According to recent studies, a good proxy of the isotropy boundary can also be determined by measuring either the maximum of precipitated proton energy flux (e.g. the DMSP b2i boundary; see Newell et al. (1998) or by observing the latitudinal profile of the hydrogen auroral emission from the ground stations (optical b2i), by Donovan et al. (2003). As discussed by Newell 

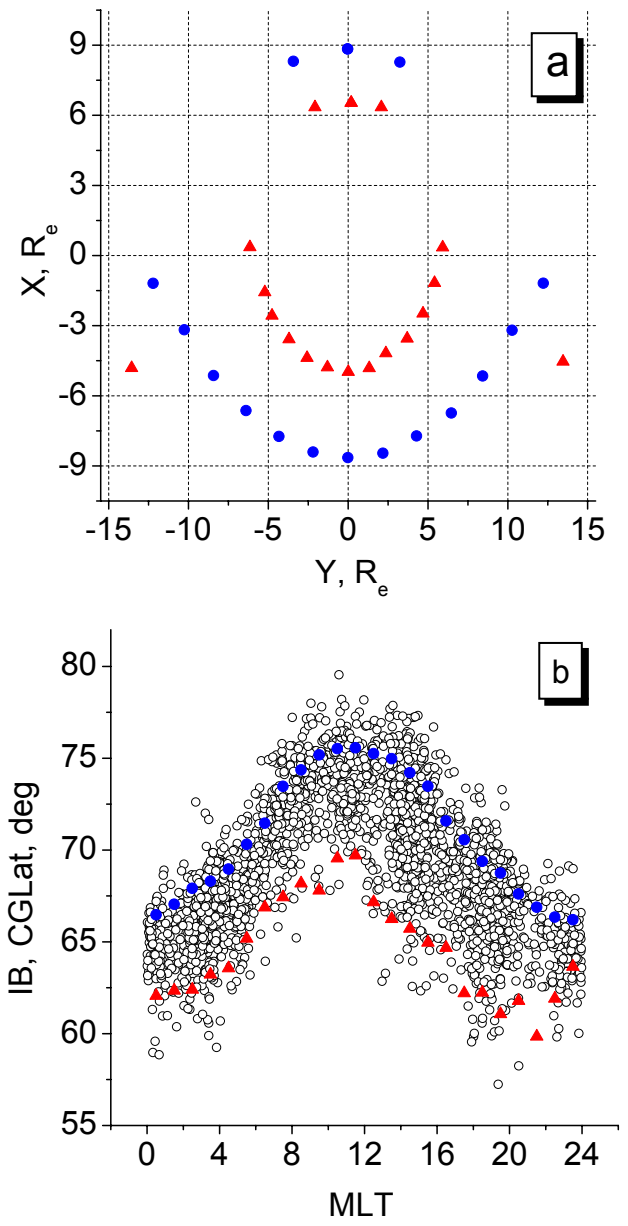

Fig. 1. (a) Proton isotropy boundaries in the equatorial magnetosphere computed from the Tsyganenko-2001 model (with $R c / \rho \sim 8)$ for $80 \mathrm{keV}$ protons. Circles denote quiet conditions (model parameters: $\mathrm{Pd}=1 \mathrm{nPa}, D_{s t} *=-5 \mathrm{nT}, B_{y}=0.5 \mathrm{nT}$, $B_{z}=1 \mathrm{nT}, \mathrm{V}=400 \mathrm{~km} / \mathrm{s}$ ), triangles denote disturbed conditions (model parameters: $\mathrm{Pd}=8.5 \mathrm{nPa}, D_{s t} *=-150 \mathrm{nT}, B_{y}=0.5 \mathrm{nT}$, $B_{z}=-11 \mathrm{nT}, \mathrm{V}=400 \mathrm{~km} / \mathrm{s}$ ). (b) Distribution of all observed isotropy boundary crossings (open circles). Blue circles and red triangles indicate average IB positions during quiet $\left(D_{s t}>-10 \mathrm{nT}\right.$, $\left.B_{z}>0 \mathrm{nT}, \quad \mathrm{Pd}<2 \mathrm{nPa}\right)$ and disturbed $\left(-200<D_{s t}<-150 \mathrm{nT}\right.$, $\left.-10<B_{z}<-5 \mathrm{nT}, 5<P_{d}<9 \mathrm{nP}\right)$ conditions.

et al. (1998) the dawn-dusk asymmetry of the b2i daily variation is probably related to the corresponding asymmetry of geosynchronous magnetic field which shows larger depression of equatorial $B_{z}$ at dusk than at dawn.

The shape of IB, being dependent on the distribution of the magnetosphere magnetic field, can be influenced by changes in a few magnetosphere current systems, including the magnetopause current, the ring current, the partial ring current and the tail current. The present work is stimulated by a growing interest in the isotropy boundary as an important diagnostic parameter and aims to provide a detailed statistical study of the global IB shape and of its controlling parameters. Using a large data set (one year-long data set obtained from two polar spacecraft), we were able to investigate the relative importance of different solar wind and activity parameters, and, taking advantage of this knowledge, to construct the empirical formula describing the dynamical proton isotropy boundary. Based on this empirical formula we then compare the calculated (from magnetospheric model) and observed boundaries.

\section{Statistical investigation of the IB}

\subsection{The data set}

We used the database of isotropic boundaries determined from comparison of precipitated (near the center of the loss cone), and trapped (pitch angle close to $90^{\circ}$ ) fluxes. Proton fluxes at energies $\mathrm{E}=80-250 \mathrm{keV}$ were observed by the identical MEPED instruments on board two polar-orbiting $(h \sim 850 \mathrm{~km})$ spacecrafts, NOAA-6 and TIROS. We used the 12-months-long data set (between July 1979 and June 1980). The observed IB positions (see the criterion of IB identification in SG95) were complemented by the hourly-averaged solar wind parameters $\left(B_{z}, B_{y}\right.$, Pd from OMNI data set) and by the activity indices $\left(D_{s t}, A E\right)$. We also computed a "merging electric field", $\varepsilon 3=V B \sin 3(\Theta / 2)$, based on solar wind data ( $V$ is the solar wind velocity, $B$ is the interplanetary magnetic field and $\Theta$ is the clock angle of IMF). Our full database consists of 25200 isotropy boundaries, which cover all MLT hours. All detected IBs are plotted in Fig. 1 . In our investigation we used the observations in both Southern and Northern Hemispheres, so the CGLat absolute values are plotted here.

\subsection{Investigation of snapshots of the IB shape}

As illustrated in Fig. 1b (and noticed in SG95), the MLT dependence of isotropy boundary latitude (IBlat) in zero approximation can be well described by a simple COS function (with MLT given in hours), i.e.:

I Blat $=A_{0}-A_{1} \cos \left[\pi\left(M L T-A_{2}\right) / 12\right]$.

The relative importance of the different parameters can be probed as follows. We can estimate the IB shape and obtain near-simultaneous "snapshots" of the isotropy boundary in the case when four nearly-simultaneous crossings of the auroral oval by two spacecraft are available. As shown in Fig. 2a each spacecraft crossing the auroral oval in one hemisphere at different MLTs gives two IB point which are separated in universal time by roughly $10 \mathrm{~min}$, one after another. Trading between the small amount of the exactly simultaneous crossings by two spacecraft and the need to avoid large configurational changes in the magnetosphere possible on the substorm time scale $(\sim 1 \mathrm{~h})$ we finally considered the observations made within a $20 \mathrm{~min}$ time slot. Besides, the nearly homogeneous MLT coverage was also required for each subset. Under these conditions we obtained 330 subsets consisting of four nearly-simultaneous IB latitudes and corresponding MLTs. Then we fit the data of each subset by a cosine 


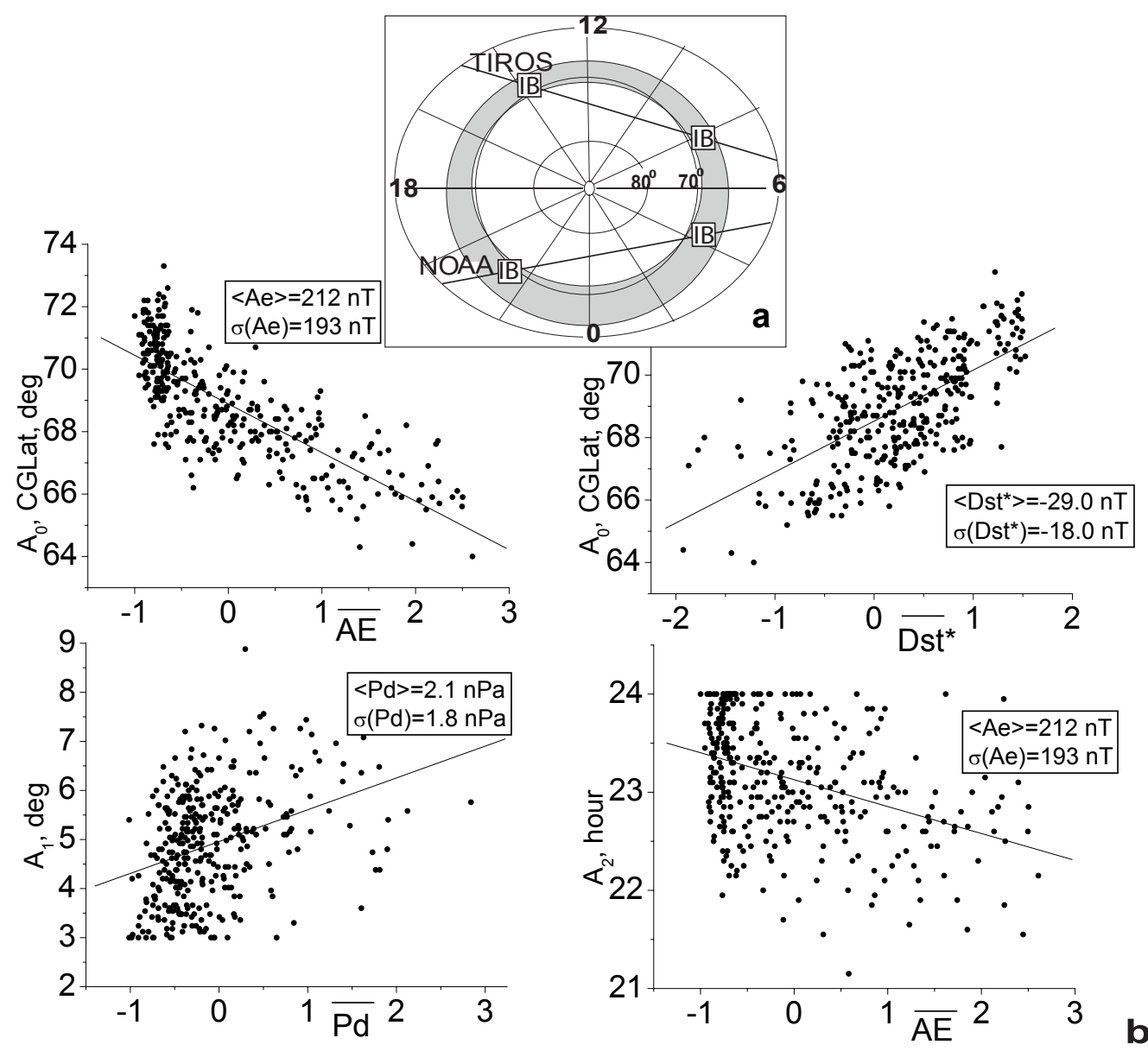

Fig. 2. (a) Scheme of simultaneous crossings of the auroral oval by two spacecraft. (b) Most significant (see Table 1) dependences of IB shape coefficients on the normalized solar wind and activity parameters. Average values and standard deviations are also shown.

function. The best fitting parameters of the cosine Eq. (2) have been determined for each subset and tagged with corresponding solar wind and activity parameters. If individual crossings fell into different UT-hours, we averaged the solar wind and activity parameters between these two hours. The corrected $D_{s t}{ }^{*}$ was computed to eliminate the contribution of the Chapman-Ferraro current system in the $D_{s t}$-index as $D_{s t}^{*}=0.8 D_{s t}-13 \sqrt{P d}$ (following Tsyganenko et al. (2002), with $D_{s t}$ expressed in $\mathrm{nT}$ and $\mathrm{Pd}$ in $\mathrm{nPa}$ ).

Having snapshots of an instantaneous IB shape we studied which solar wind and activity parameters control each of the 3 coefficients $\left(A_{0}, A_{1}, A_{2}\right)$ in Eq. (2), using correlation and regression analysis. To facilitate the comparisons of the parameters' influence we used their normalized form computed as $\bar{P}=(P-<P>) / \sigma(P)$, where $<P>$ and $\sigma(P)$ are the average value and standard deviation, correspondingly. With such normalization the coefficients of the linear regression also reflect how much the coefficients are affected by different controlling parameters. The values of the correlation/regression coefficients (CC/RC) are presented in Table 1; the most important results are also illustrated in Fig. 2 b.

The correlation/regression coefficients show a clear hierarchy of parameter influence. The most ordered is the equator- ward shift $\left(A_{0}\right)$, with the strongest influence of the $A E$ index variations $(\mathrm{CC}=-0.78, \mathrm{RC}=-1.61)$, the next is the $D_{s t} *$ in$\operatorname{dex}(0.64 / 1.58)$, then the merging rate $\epsilon 3(-0.61 /-1.35)$ and IMF $B_{z}(0.59 / 1.24)$. (Note that all three display a strong mutual correlation, see Table 2.) Figure $2 \mathrm{~b}$ demonstrates that the increase of magnetosphere activity (i.e. the $A E$ increase from $50 \mathrm{nT}$ to $900 \mathrm{nT}$ and the $D_{s t} *$ decrease from $25 \mathrm{nT}$ to $-60 \mathrm{nT}$ ) leads to the shift of the IB shape towards the low latitudes by $\sim 8 \mathrm{deg}$. The less ordered is the daily variation amplitude ( $A_{1}$, maximal correlation coefficient less than 0.36 ), which shows contributions from $\mathrm{Pd}, B_{z}$, and $D_{s t} *$ (the largest regression coefficient is for $\mathrm{Pd}, \mathrm{RC}=0.65$ ). The increase of Pd from $0.5 \mathrm{nPa}$ to $8.5 \mathrm{nPa}$ results in the increase in the daily variation amplitude by $\sim 4 \mathrm{deg}$. As discussed below, Pd has an essential influence on the daily variation, despite its a weak correlation with $A_{1}$. The phase shift in MLT $\left(A_{2}\right)$ is mostly influenced by mutually related parameters $A E$ $(-0.41 /-0.27)$ and IMF $B_{z}(0.37 / 0.26)$, but, as will be shown below, $B_{z}$ does not greatly improve the representation of the dawn-dusk asymmetry. The increase in the $A E$-index from $50 \mathrm{nT}$ to $900 \mathrm{nT}$ leads to the dusk shift of the IB shape by approximately $2 \mathrm{~h}$. The IMF $B_{y}$ influence was not observed for any parameter of the cosine fit. 
Table 1. Correlation and regression coefficients between the coefficients of cosine fit and normalized activity parameters for 330 IB snapshots.

\begin{tabular}{rrrrrrrrrrrrrr}
\hline & \multicolumn{1}{c}{$\mathrm{Pd}$} & \multicolumn{2}{c}{$B_{y}$} & \multicolumn{2}{c}{$B_{z}$} & \multicolumn{2}{c}{$\epsilon 3$} & \multicolumn{2}{c}{$D_{s t} *$} & \multicolumn{2}{c}{$A E$} \\
\hline & $\mathrm{CC}$ & $\mathrm{RC}$ & $\mathrm{CC}$ & $\mathrm{RC}$ & $\mathrm{CC}$ & $\mathrm{RC}$ & $\mathrm{CC}$ & $\mathrm{RC}$ & $\mathrm{CC}$ & $\mathrm{RC}$ & $\mathrm{CC}$ & $\mathrm{RC}$ \\
$\mathrm{A} 0$ & -0.20 & -0.56 & -0.33 & -0.76 & 0.59 & 1.24 & -0.61 & -1.35 & 0.64 & 1.58 & -0.78 & -1.61 \\
$\mathrm{~A} 1$ & 0.36 & 0.65 & -0.17 & -0.24 & 0.23 & 0.31 & -0.13 & -0.20 & -0.18 & -0.29 & -0.12 & -0.15 \\
$\mathrm{~A} 2$ & 0.07 & 0.07 & -0.19 & -0.15 & 0.37 & 0.26 & -0.34 & -0.25 & 0.19 & 0.16 & -0.41 & -0.27 \\
\hline
\end{tabular}

Table 2. Mutual correlations between different interplanetary parameters and activity indexes for the data set of 330 IB snaphot.

\begin{tabular}{crrrrr}
\hline & $D_{s t} *$ & $A E$ & $B_{z}$ & $\epsilon 3$ & $B_{y}$ \\
$D_{s t} *$ & & & & & \\
$A E$ & -0.47 & & & & \\
$B_{z}$ & 0.32 & -0.63 & & & \\
$\mathrm{e} 3$ & -0.41 & 0.71 & 0.84 & & \\
$B_{y}$ & 0.15 & 0.10 & 0.04 & 0.03 & \\
$\mathrm{Pd}$ & 0.01 & 0.25 & 0.08 & 0.30 & 0.06 \\
\hline
\end{tabular}

\section{Nonlinear-regression empirical model of the proton isotropy boundary}

\subsection{Empirical expression of the IB shape}

Taking advantage of knowing the hierarchy of basic controlling parameters, we then expand the $A_{0}, A_{1}$ and $A_{2}$ coefficients as a function of the most important controlling parameters, namely, we represent $A_{0}$ and $A_{2}$ by the linear function of original (non-normalized) $A E$ and $D_{s t}{ }^{*}$ : $A_{0}=a_{0}+a_{1} \mathrm{AE}+a_{2} D_{s t}^{*} ; A_{2}=a_{5}+a_{6} A E ; A_{1}$ by the root function of non-normalized $\mathrm{Pd}: A_{1}=a_{3}+a_{4} \sqrt{P d}$. In this stage we excluded the merging electric field and $B_{z}$ IMF from analysis, because $\varepsilon 3$ has a large correlation with the $A E$-index, and $B_{z}$ has a small correlation and small regression coefficient with $A_{0}, A_{1}$ and $A_{2}$ (Table 1). Our function is of the form:

$$
\begin{aligned}
& I B f i t=a_{0}+a_{1} A E+a_{2} D_{s t}^{*}+\left(a_{3}+a_{4} \sqrt{P d}\right) \times \cos \\
& \left(\left(M L T+a_{5}+a_{6} A E\right) \pi / 12\right)
\end{aligned}
$$

Using the nonlinear, multi-parametric least-square regression procedure and our entire database of proton isotropy boundaries (consisting of 25200 samples), we determined the best fit coefficients: $a_{0}=71.33^{\circ}$ CGLat $a_{1}=-0.0057^{\circ} / \mathrm{nT} ; a_{2}=0.047^{\circ} / \mathrm{nT} a_{3}=-3.23^{\circ}$ CGLat; $a_{4}=-0.99^{\circ} / \mathrm{nPa} ; a_{5}=0.37 \mathrm{~h} ; a_{6}=0.0016 \mathrm{~h} / \mathrm{nT}$. We then compare the observed isotropy boundaries, IBobs, with IBfit predicted from Eq. (3). As one can see in Fig. 3 they are in good agreement: the correlation coefficient is $\mathrm{CC}=0.91$ and the standard deviation is $1.60^{\circ}$.

After the function was built we also tried to estimate its eligibility: we tried different parameter sets for our function.

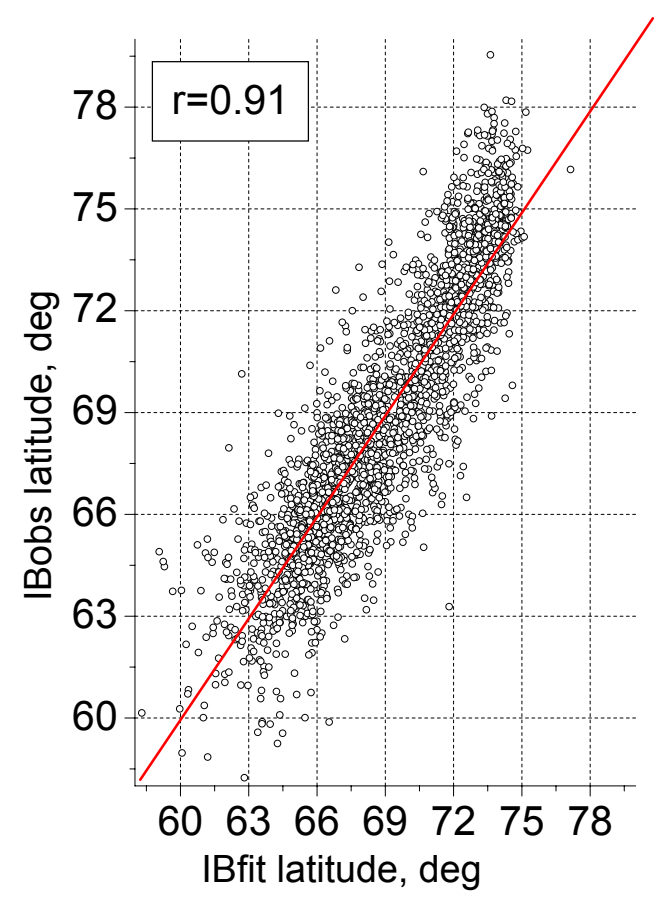

Fig. 3. Comparison between observed IB latitudes (IBobs) and those predicted using the Eq. (3) (IBfit).

First, because Pd has a weak correlation with $A_{1}(\mathrm{CC}=0.36$, $\mathrm{RC}=0.65$ ), we exclude the $\mathrm{Pd}$ from the function parameter sets. This leads to a considerable decrease in the correlation coefficient (CC falls down from 0.91 to 0.67 ) and dispersion rose to $2.23^{\circ}$. The variation of the $\mathrm{Pd}$ power law coefficient did not significantly change the correlation coefficient. Second, we expand each of the $A_{0}, A_{1}$ and $A_{2}$ coefficients as a linear function of all solar wind $\left(\mathrm{Pd}, B_{y}, B_{z}\right)$ and activity parameters $\left(\varepsilon 3, D_{s t}{ }^{*}, A E\right)$, but finally left those given in Eq. (3) after learning that the addition of the new terms does not improve the fit quality. And finally, we tried a dipole tilt as a parameter of the function but failed to reveal any pronounced effect.

3.2 Comparison with isotropy boundaries predicted from Tsyganenko-2001 model

An empirical formula for the isotropy boundary Eq. (3) can be compared with the predictions made from the 
recent empirical magnetospheric model T01. This model is the first empirical model in which the partial ring current and the dawn-dusk asymmetry were taken into account explicitly (Tsyganenko et al., 2002). We assigned the input parameters of the model (Pd, $\left.D_{s t}, B_{y}, B_{z}, \mathrm{~V}\right)$ to simulate both quiet and disturbed conditions and traced the field lines, beginning from different meridians and latitudes, to find the equatorial points in the nightside magnetosphere and points in the dayside outer cusp where condition $R_{c} / \rho=8$ is satisfied for $80 \mathrm{keV}$ protons. We then found the ionospheric projections (CGLat, MLT) of these field lines, the model isotropy boundaries (IBmod).

To calculate the empirical IB for these parameters, we had to modify Eq. (3) because the $A E$-index is not among the input parameters of the T01model. Because the $A E$-index has a high correlation with $\varepsilon 3$ ( $\mathrm{RC}=0.71)$, we substituted the $A E$-index with the $\varepsilon 3$ (which is the function of $V$ and $B$ ) in the fitting Eq. (3):

$$
\begin{aligned}
& I B f i t=a_{0}+a_{1} \varepsilon 3+a_{2} D s t^{*}+\left(a_{3}+a_{4} \sqrt{P d}\right) \times \cos \\
& \left(\left(M L T+a_{5}+a_{6} \varepsilon 3\right) \pi / 12\right) .
\end{aligned}
$$

Repeating the multiple regression we found new fitting coefficients $a_{0}=71.21^{\circ} \mathrm{CGLat} ; a_{1}=-0.82^{\circ}(\mathrm{mV} / \mathrm{m})$; $a_{2}=0.058^{\circ} \mathrm{nT} ; \quad a_{3}=-3.23^{\circ} \mathrm{CGLat} ; \quad a_{4}=-0.98^{\circ} \mathrm{nPa}$; $a_{5}=0.51 \mathrm{~h} ; a_{6}=0.20 \mathrm{~h}(\mathrm{mV} / \mathrm{m})$. For comparison, the correlation coefficient between observed IBobs latitudes and empirical IBfit derived from Eq. (4) is $\mathrm{RC}=0.89$, the standard deviation is $1.73^{\circ}$.

Comparisons between the isotropy boundary predicted from the T01 model (IBmod) and empirical isotropy boundaries Eq. (4) are shown in Fig. 4, for both quiet $(\mathrm{Pd}=1 \mathrm{nPa}$, $\mathrm{V}=400 \mathrm{~km} / \mathrm{s}, D_{s t} *=-5 \mathrm{nT}$, IMF $\left.B_{z}=1 \mathrm{nT}, B_{y}=0.5 \mathrm{nT}\right)$ and very disturbed $\left(\mathrm{Pd}=8 \mathrm{nPa}, \mathrm{V}=400 \mathrm{~km} / \mathrm{s}, D_{s t} *=-150 \mathrm{nT}\right.$, IMF $B_{z}=-11 \mathrm{nT}, B_{y}=0.5 \mathrm{nT}$ ) conditions.

As seen in the Fig. 4, on the nightside the agreement between model predictions and empirical IBfit is very good for the quiet state. However, the IBmod shape is considerably distorted during very disturbed conditions, showing the large deviation from the cosine shape and the $\sim 4^{\circ}$ latitudinal difference from observation near the midnight. We also see the large disagreement between IBmod and IBfit for both magnetospheric states on the dayside $\left(\sim 2^{\circ}\right.$ CGLat for quite state and $\sim 3^{\circ}$ CGLat for disturbed state). On the other hand, the model reveals the dawn-dusk asymmetry as the shift in the latitude minimum by $\sim 2 \mathrm{~h}$ MLT duskward, in agreement with the empirical-based results.

\section{Conclusions}

In this paper we have investigated the variations of the isotropy boundary of the energetic protons $(\mathrm{E}=80-250 \mathrm{keV})$. Using the large data set consisting of 25200 IB determinations we studied how the isotropy boundary shape (the dependence of the IB latitude on the magnetic local time, MLT) depends on the interplanetary parameters $\left(B_{z}, B_{y}, \mathrm{Pd}, \varepsilon 3\right)$
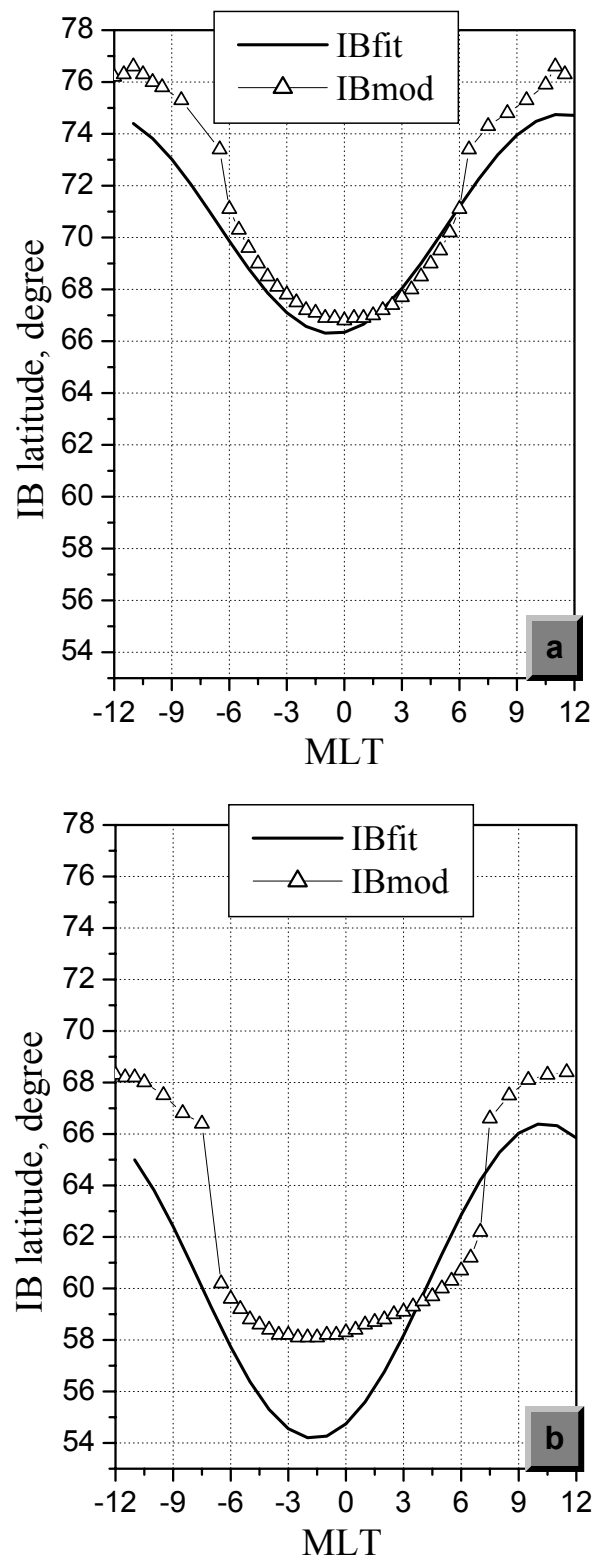

Fig. 4. Comparisons between the isotropic boundaries predicted from the T01 model (IBmod, open triangle) and obtained from empirical Eq. (4) (IBfit, solid line) for quiet (a, $\mathrm{Pd}=1 \mathrm{nPa}, D_{s t} *=-5 \mathrm{nT}$, $\left.B_{y}=0.5 \mathrm{nT}, B_{z}=1 \mathrm{nT} \mathrm{V}=400 \mathrm{~km} / \mathrm{s}\right)$ and disturbed $(\mathrm{b}, \mathrm{Pd}=8.5 \mathrm{nPa}$, $D_{s t} *=-150 \mathrm{nT}, B_{y}=0.5 \mathrm{nT}, B_{z}=-11 \mathrm{nT} \mathrm{V}=400 \mathrm{~km} / \mathrm{s}$ ) conditions.

and activity indexes $\left(A E, D_{s t} *\right)$. It was shown that different indexes (and accordingly the different large-scale current systems) affect the IB shape behavior by different ways (see Table 1 and Fig. 2). We specified the empirical function that predicts the isotropy boundary based on the solar wind data, $D_{s t} *$ and $A E$ indexes and compared it with the isotropy boundary calculated from Tsyganenko-2001 model.

Based on the assumption that changes in the isotropy boundary shape reflect the corresponding changes in the magnetospheric magnetic field, the interpretation of these results could be as follows. 
1. The increase in the magnetosphere convection shifts of the IB(MLT) curve. Such a shift is the result of the overall depression of the inner-magnetospheric equatorial magnetic field, which increases with the growth of the tail and both the symmetric and partial ring currents reflected by the increase of $A E$ and $D_{s t} *$. Indeed, according to Eq. (1) for the particle of fixed energy $(G=$ const $)$, the decrease of magnetic field $B_{z^{-}}$ component results in the earthward shift of the equatorial isotropy boundary (see Fig. 1a).

2. The daily amplitude $\left(A_{1}\right)$ is controlled mostly by the solar wind dynamic pressure. This correlation arises from the earthward displacement and intensification of both the magnetopause and tail current, which increases the noon-midnight asymmetry of equatorial magnetic field (see e.g. the analysis of the magnetic field at $6.6 R_{e}$ made by Rufenach et al. (1992).

3. Finally, the magnetospheric activity increase also increases the duskward phase shift $\left(A_{2}\right)$ of the IB curve. This effect can be explained by the dawn-dusk asymmetry of the magnetic field in the near-Earth magnetosphere by the larger increase in the field depression at dusk when the $A E$ increases. The dawn-dusk asymmetry in the inner magnetic field is mostly related to the partial ring current (Tsyganenko et al., 2002), so the fact of better control of the dawn-dusk shift of the isotropy boundary by the $A E$ index than by $D_{s t}$ * indicates that the dawn-dusk asymmetry of the magnetic field is caused by the partial ring current.

These dependencies are consistent with previous results. For example, the amount of the IB phase shift (1.5-2 h MLT in disturbed time) is consistent with statistical results obtained by (Donovan et al. (2003) (see their Fig. 4). Conclusions that the equatorward shift and MLT shift depend mostly on the $A E$-index and that the daily variation amplitude is mainly controlled by Pd are consistent with the conclusions of SG95.

The basic result of comparison with model prediction is that in the quiet conditions they agree well on the nightside, whereas large differences are found during magnetic storms. Such differences can be explained in several ways. First of all, we have to take into account the possible deficiencies of each magnetospheric model (how well it describes the distribution of $B_{z}$ and the electric current in the equatorial region), arising due to insufficient coverage of real and parameter space with observations, or due to insufficient flexibility of the functions describing the current systems and their parameter dependence. Our results then indicate that the magnetic field depression in the inner magnetosphere is deeper than that predicted by the T01 model. A second reason can the enhanced wave activity, which provides the additional pitchangle scattering in the inner region. Typically, this scattering is weak and produces very anisotropic fluxes in the loss cone (called the low-latitude proton precipitation, LLPP, Gvozdevsky et al., 1997), however, during the storm it can strongly intensify and provide almost isotropic distributions. In that case it can be difficult to distinguish IB and LLPP boundaries and the equatorial boundary of isotropic precipitation can be erroneously defined a few degrees equatorward from the true boundary of adiabatic/nonadiabatic motion in the equatorial magnetosphere. Finally, the disagreement during magnetic storms may arise because the cosine function is not appropriate for these conditions. To clarify which of the abovementioned reasons give the major contribution to the discussed discrepancy the additional investigations are required.

Acknowledgements. The NOAA spacecraft data (H. Sauer was a principal investigator of MEPED instrument) have been made available through WDC-A in Boulder, we thank P. Newell for his help in providing these data. This work was supported by RFBR grant 04-05 64932 as well as by RF President grant NSH-760.2003.5)

Topical Editor T. Pulkkinen thanks N. Ganushkina for her help in evaluating this paper.

\section{References}

Donovan, E. F., Jackel, B. J., and Voronkov, I. et al.: Groundbased optical determination of the b2i boundary: A basis for an optical MT-index, J. Geophys. Res., 108(A3), 1115, doi:10.1029/2001JA009198, 2003.

Gvozdevsky B. B., Sergeev V. A., and Mursula K.: Long lasting energetic proton precipitation in the inner magnetosphere after substorms, J. Geophys. Res., 102(A11), 24 333-24 338, 1997.

Newell P. T, Sergeev V. A., Bikkuzina G. R., and Wing S.: Characterizing the state of the magnetosphere: Testing the ion precipitation maxima latitude (b2i) and the ion isotropy boundary, J. Geophys. Res., 103(A3), 4739-4745, 1998.

Rufenach C. L., McPherron R. L., and Schaper J.: The quiet geomagnetic field at geosynchronous orbit and its dependence on solar wind dynamic pressure, J. Geophys. Res., 97(A1), 25-42, 1992.

Sergeev V. A., Sazhina, E. M., Tsyganenko, N. A., Lunbland, J. A., and Soraas F.: Pitch angle scattering of energetic protons in the magnetotail current sheet as the dominant source of their isotropic presipitation into the nigtside ionosphere, Planet Space Sci., 31, 1147-1158, 1983.

Sergeev V. A. and Malkov M. V.: Diagnostic of the magnetic configuration of the plasma sheet from measurements of energetic electrons above the ionosphere, Geomagn. Aeron., 28, 649-653, 1988.

Sergeev V. A, Malkov M. V., and Mursula K.: Testing the isotropic boundary algorithm method to evaluate the magnetic field configuration in the tail, J. Geophys. Res., 98, 7609-7620, 1993.

Sergeev V. A. and Gvozdevsky B. B.: MT-index: a possible mew index to characterize the magnetic configuration of the magnetotail, Ann. Geophys., 13, 1093-1103, 1995.

Sergeev V. A., Bikkuzina G. R., and Newell P. T.: Dayside isotropic precipitation of energetic protons, Ann. Geophys., 15, 12331245, 1997.

Tsyganenko N. A.: A model of the near magnetosphere with a dawn-dusk asymmetry, J. Geophys. Res., 107,SMP 12-1, doi:10.10292001JA00021922001, 2002.

West H. I., Buck R. M., and Kivelson M. G.: On the configuration of the magnetotail near midnight during quiet and weakly disturbed periods: Magnetic field modeling, J. Geophys. Res., 83(A8), 3819-3828, 1978. 